\title{
Körperliche Aktivität schützt Herz und Gefäße auch nach Brustkrebs
}

In einer prospektiven Analyse von Daten aus 2 registerbasierten Kohortenstudien wurde der Frage nachgegangen, ob körperliche Aktivität wie in der Allgemeinbevölkerung auch das Risiko für kardiovaskuläre Erkrankungen (CVD) bei Brustkrebspatientinnen reduziert.

n die Auswertung wurden 2.973 bei Diagnose eines nicht metastasierten Mammakarzinoms herz- und gefäßgesunde Frauen (mittleres Alter 53 Jahre) nach Abschluss der adjuvanten Therapie aufgenommen. Sie hatten angegeben, wie viel Zeit sie in ihrer Freizeit mit körperli-

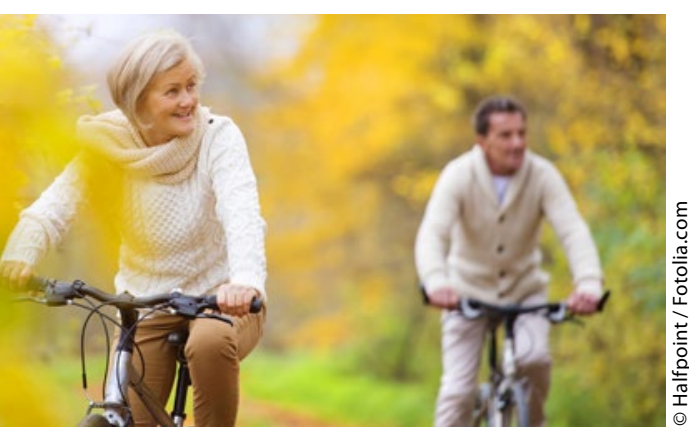

Auch bei Brustkrebspatientinnen schützt regelmäßige Bewegung das Herz. cher Betätigung verbrachten. Die Aktivität wurde in metabolische Äquivalente (MET) in Stunden pro Woche umgerechnet und in 4 Kategorien von $<2$ MET-h/ Woche bis $\geq 25$ MET-h/Woche eingeteilt. Primärer Endpunkt war das erste Auftreten eines der folgenden Endpunkte: Erstdiagnose einer koronaren Herzerkrankung (KHK), einer Herzinsuffizienz, eines Herzklappendefekts, einer Herzrhythmusstörung, eines Schlaganfalls oder ein kardiovaskulär bedingter Tod nach Studieneintritt. Die mediane Beobachtungszeit betrug 8,6 Jahre.

In der multivariablen Analyse nahm die Inzidenz kardiovaskulärer Ereignisse mit steigender MET-Kategorie ab $\left(\mathrm{p}_{\text {trend }}<0,001\right)$. Verglichen mit der niedrigsten Kategorie (<2 MET-h/Woche) betrug die adjustierte Hazard Ratio bei 2-10,9 MET-h/Woche 0,91 (95\%-Konfidenzintervall [95\%-KI] 0,76-1,09), bei
11-24,5 MET-h/Woche 0,79 (95\%-KI $0,66-0,96)$ und bei $\geq 24,5$ MET-h/Woche 0,65 (95\%-KI 0,53-0,80). Ähnliche Trends wurden für die Inzidenz der KHK und der Herzinsuffizienz festgestellt (p jeweils $<0,05$ ).

Die in den USA empfohlene Intensität körperlicher Aktivität bei erwachsenen Krebspatienten von $\geq 9$ MET-h/Woche war verglichen mit $<9$ MET-h/Woche assoziiert mit einer adjustierten Risikoreduktion eines kardiovaskulären Ereignisses von $23 \%(\mathrm{p}<0,001)$. Die Assoziation von Aktivität und CVD-Inzidenz war in allen Altersgruppen feststellbar und unabhängig von bereits bestehenden CVDRisikofaktoren, dem Menopausestatus bei Diagnose oder der Art der Therapie.

Fazit: Körperliche Aktivität kann das kardiovaskuläre Risiko von Frauen mit nicht metastasiertem Brustkrebs deutlich und unabhängig von Alter und Therapie senken.

Friederike Klein

Jones JA et al. Exercise and Risk of Cardiovascular Events in Women With Nonmetastatic Breast Cancer. J Clin Oncol. 2016;34(23):2743-9.

\section{Sicherheit von Everolimus plus Exemestan bei Brustkrebs erneut geprüft}

Hinsichtlich der Sicherheit sind die Ergebnisse einer europäischen Phase-IlllbStudie bei Patientinnen mit Hormonrezeptor-positivem, HER2-negativem fortgeschrittenem Brustkrebs vergleichbar mit denen der BOLERO-2-Studie.

$P^{\circ}$ ostmenopausale Patientinnen mit vorangegangener Aromatasehemmer-Therapie hatten ein Rezidiv oder einen Progress entwickelt und erhielten im Rahmen der offenen Sicherheits-Studie BALLET Everolimus (EVE) plus Exemestan (EXE). Die mediane Behandlungsdauer betrug 5,1 Monate für EVE und 5,3 Monate für EXE. Bei 65,1\% war dies bereits die 3. oder eine noch spätere Therapielinie. Für die Analyse der unerwünschten Ereignisse (AE) konnten Daten von 2.131 Patientinnen einfließen. Bei $81,8 \%$ der Frauen wurden AE in Zusammenhang mit einem der beiden oder beiden Therapeutika dokumentiert, bei 27,2\% der Fälle vom Schweregrad 3/4.

Die häufigsten nicht hämatologischen AE waren Stomatitis (insgesamt 52,8\%, EVE-abhängig 50,8\%) und Asthenie $(22,8 \% / 14,6 \%)$. Häufigste hämatologische Ereignisse waren Anämie (14,4\% insgesamt, 8,1\% EVE-abhängig) und Thrombozytopenie (5,9\%/4,6\%). Über 70 -jährige Patientinnen brachen öfter die Therapie ab als jüngere (23,8 vs. $13,0 \%)$.

Altersunabhängig war die Inzidenz EVE-assoziierter AE bei Erstlinientherapie geringer als in späteren Therapielinien. Die AE-Inzidenz war unabhän- gig vom Body-Mass-Index. Insgesamt erlitten $8,5 \%$ der Patientinnen mindestens einmal ein EVE-abhängiges schweres AE. 121 Patientinnen (5,7\%) verstarben. Der Tod von 66 Patientinnen (3,1\%) wurde auf einen Krankheitsprogress zurückgeführt, bei 46 Frauen $(2,2 \%)$ wurde vermutet, dass der Tod mit der EVETherapie zusammenhängt.

Fazit: In der größten bisher berichteten Sicherheitskohorte, die auch stark vorbehandelte und ältere Patientinnen einschloss, bestätigte sich das aus der Studie BOLLERO-2 bekannte Sicherheitsprofil von EVE plus EXE. Friederike Klein

Jerusalem G et al. Safety of everolimus plus exemestane in patients with hormone-receptor-positive, HER2-negative locally advanced or metastatic breast cancer progressing on prior non-steroidal aromatase inhibitors: primary results of a phase IIIb, open-label, single-arm, expanded-access multicenter trial (BALLET). Ann Oncol. 2016;27(9):1719-25. 